\title{
Systemtheorie und Rational Choice Theorie ${ }^{1}$
}

\author{
Rudolf Stichweh \\ Universität Bielefeld, Fakultät für Soziologie, PF 1001 31, D-33501 Bielefeld
}

Zusammenfassung: Der Aufsatz identifiziert ein Parsonianisches Paradigma in der Entwicklung der Disziplin Soziologie, das einen individualistisch interpretierten Rationalitätskern des Handelns einbettet in eine soziale Umwelt aus Normen, Werten und Institutionen und dann die Relation dieser beiden Seiten zu präzisieren versucht. Diese duale Denkstruktur scheint zunächst die Entwicklung der Systemtheorien und der Rational Choice Theorien bestimmt zu haben. Die Systemtheorie löst sich aber seit Parsons' Professionstheorie, die Rationalität als geschichtsabhängige gesellschaftliche Institution deutete, und später dann mit dem Umbau in Richtung auf eine Kommunikations- und eine Unterscheidungstheorie von dieser Ausgangssituation ab. In der Folge vergleicht der Aufsatz strategisch relevante Theorieentscheidungen der beiden Theorien in der Gegenwart: a) die Frage der Stabilität von Präferenzen vs. einen Sozialkonstruktivismus, der sich gerade für die Entstehung von Präferenzen und für die soziale Konstitution von Akteuren interessiert; b) den Umgang mit differenzlosen Begriffen am Beispiel des Rationalitätsbegriffs; c) den Status von "utilities“ und Zwecken im Rational Choice Denken im Vergleich zu einer Theorie, die diese als Zurechnungsentscheidungen denkt; d) den beiden Theorien gemeinsamen Interessenschwerpunkt, der mit Phänomenen des generalisierten Tauschs und der medienvermittelten Kommunikation zu tun hat; e) den strategischen Stellenwert der Mikro-Makro-Unterscheidung als Prüfstein für eine Theorie. Am Ende steht die Vermutung, daß die Differenz der beiden Theorien durch die Differenz ihrer interdisziplinären Milieus stabilisiert wird.

\section{Talcott Parsons und die Differenzierung von Systemtheorie und Rational Choice Theorie}

In den dreißiger Jahren hat die Konfrontation von Systemtheorie und Rational Choice Theorie einen Wendepunkt der Soziologiegeschichte markiert. Talcott Parsons geht 1937 in ,The Structure of Social Action" von einer Situation aus, die er durch eine Dominanz biologischen und ökonomischen Denkens charakterisiert sieht. Während die Biologie die für den Handelnden nicht verfügbaren Aspekte des Handlungsgeschehens beschreibt, thematisiert die Ökonomie seine Handlungswahl. Der Handelnde wird als ein Beobachter gesehen, der in Hinsicht auf die von ihm verfolgten Ziele die für diese Ziele angemessenen Mittel dank einer gleichsam . wissenschaftlichen Beobachtung seiner Situation wählt und in dieser Hinsicht rational ist. Diese Theorie der Rationalität hat Parsons 1937 noch nicht kritisiert. ${ }^{2}$ Parsons schwankt, ob er dem Handelnden ein wissenschaftlich vollständiges Kausalwissen hinsichtlich der für die Mittel-

\footnotetext{
1 Revidierte Fassung der Antrittsvorlesung an der Fakultät für Soziologie der Universität Bielefeld am 7. Juni 1995. Danken möchte ich den Mitgliedern eines von Hartmut Esser initiierten Arbeitskreises, in dessen Rahmen dieser Text am 30. Juni 1995 in der Universität Leipzig diskutiert wurde.

${ }^{2}$ Siehe dazu näher Stichweh 1980.
}

wahl relevanten Aspekte der Situation oder nur ein praktisch adäquates Handlungswissen zuschreiben soll. D.h., der Rationalitätsbegriff bleibt unentschieden hinsichtlich der Frage, ob er näher an den Wahrheitsansprüchen der Wissenschaft oder an den Funktionszusammenhängen der Technik ist. ${ }^{3}$ Aber diese Diskussion tangiert Parsons' Einverständnis mit den Rationalitätsunterstellungen der Ökonomie nicht.

Sein Einwand war 1937 ein anderer: Die ökonomisch-biologische Auffassung des Handelns erlaubt nicht zu sehen, daß und inwiefern Handeln ein System ist. Die rationale Wahl von Mitteln für gegebene Zwecke erfaßt nur einen unteren Sektor des Handlungsgeschehens. Es gibt aber darüberliegende Sektoren des Handelns, in denen Ziele mittels von Normen zu systematischen Zusammenhängen geordnet werden und in denen weiterhin Normen auf der Basis von Werten Selektionsprozessen unterliegen. Diese Hierarchie von Mitteln, Zielen, Normen und Werten macht die Systematizität oder den Systemcharakter des Handelns aus. Eine solche systematische Ordnung auf der Basis von Normen und Werten ist einerseits - wie dies auch für die biologischen Determinanten des Handelns galt - eine Umwelt des Handelns. Aber sie ist - mit Claude Bernards Begriff - eine ,innere Umwelt" des Handelns. Sie definiert die Eigenwirklichkeit der Gesellschaft gegenüber biologi-

\footnotetext{
${ }^{3}$ Siehe dazu Parsons 1937: 65, insb. Fn. 1; 83; 369-70.
} 
schen Determinanten und individuellen rationalen Kalkülen. Und als eine solche Eigenwirklichkeit tritt sie den rationalen Kalkülen als eine nichtratıonale - nicht etwa irrationale - Bestimmungsgröße des Handelns gegenüber. Soziologie ist insofern für den frühen Parsons - und in dieser Hinsicht muß der Einfluß von Vilfredo Pareto angemessen gewürdigt werden - die Wissenschaft des Nichtrationalen. Und zum Moment der Rationalität als einer subjektiven Bestimmungsgröße des Handelns kommt jetzt das Moment der Anstrengung hinzu (Parsons sagt ,effort"), die sich der Handelnde auferlegt, um den nichtrationalen Bestimmungsgründen des Handelns - Normen und Werte -, durch die sich der Handelnde gebunden fühlt, Geltung in der Wirklichkeit des Handelns zu verschaffen. Die kollektive Eigenrealität der $\mathrm{Ge}$ sellschaft als System von Normen und Werten und die Anstrengung des einzelnen Handelnden in der Umsetzung dieser Normen und Werte sind dann offensichtlich die beiden Komponenten, die den Anspruch auf eine voluntaristische Handlungstheorie begründen. Und Voluntarismus meint damit vor allem eine Zurückdrängung jeder biologischen Determination und eine Indienstnahme rationaler Kalküle durch nichtrationale Leitgesichtspunkte.

Der Erfolg der „Structure of Social Action“ ist bekannt. Für zwei oder drei Jahrzehnte hat dies den Einfluß von Rational Choice Theorien in der Soziologie eng limitiert. ${ }^{4}$ Darüber hinaus wurde auf der Basis dieser Weichenstellung die Entwicklung der Rational Choice Theorie in eine Richtung gelenkt, die noch bis heute zu dominieren scheint. Es ist auffällig, wie sehr Rational Choice Erklärungen auch in der Gegenwart um zwei Varianten der Erklärung desselben Handlungsproblems kreisen. Entweder wollen sie demonstrieren, daß sich ein kollektives Handlungsresultat erklären läßt, ohne die Annahme institutionalisierter Normen zu Hilfe nehmen zu müssen. Dies etwa im Sinne des berühmten Coase Theorems, das für bestimmte Situationen, in denen das Handeln eines Akteurs Nachteile (i.e. Externalitäten) für andere Akteure mit sich bringt, zu zeigen versucht, daß die Hinzufügung eines wie auch immer gestalteten Rahmens rechtlicher Regulierung das unter den Akteuren auf der Basis von Marktmechanismen ohne rechtliche Regulierung ausgehandelte Handlungsergeb-

\footnotetext{
${ }^{4}$ Dies muß mit dem Vorbehalt formuliert werden, daß es Forschung über den Einfluß der "Structure of Social Action" noch so gut wie nicht gibt. So auch Camic 1989: 94, Fn. 31 .
}

nis nicht verändern würde. ${ }^{5}$ Die zweite Variante ist die, daß man den Beweis zu führen versucht, daß eine Rational Choice Theorie die Entstehung von Normen, Werten und Institutionen allein auf der Basis ihrer Grundannahmen hinsichtlich Selbstinteresse, Nutzenmaximierung, Tausch von benötigten gegen jetzt bereits kontrollierte Ressourcen etc. zu erklären imstande ist. Insofern dominiert in der Rational Choice Theorie implizite ein Parsonianisches Paradigma, das einen individualistisch interpretierten Rationalitätskern annimmt, der von Normen, Werten und Institutionen umgeben ist oder eingehegt wird, so daß sich alles Interesse auf die symmetrisch oder asymmetrisch zu interpretierende Relation dieser beiden Komplexe richtet. Ich will die scheinbare Selbstverständlichkeit dieser dualistischen Schwerpunktsetzung an dieser Stelle nur noch am Beispiel eines Aufsatzes von Michael Hechter belegen. In diesem Text von 1990 geht es um die Erklärung der Entstehung kooperativer sozialer Institutionen. In seinen einleitenden Überlegungen affirmiert Hechter die Unverzichtbarkeit einer Prämisse, die den Akteuren Selbstinteresse unterstellt. Angesichts dieser unverzichtbaren Prämisse sei es unzulässig, internalisierte Werte oder einen noch so kleinen Anteil von Altruismus zu präsupponieren, weil man sich damit zu sehr die Frage erleichtere, wie ein Akteur die Ungewißheit hinsichtlich des Handelns des anderen überwindet und wie schließlich Kooperation als die Überwindung dieser Ungewißheit möglich wird (Hechter 1990: 16 und 28, Fn. 6). Die Leitunterscheidung ist hier also Selbstinteresse vs. internalisierte Werte bzw. Altruismus, und die Wahl nur der einen Seite der Unterscheidung als einer zulässigen Prämisse wird wissenschaftstheoretisch, nicht mit Argumenten hinsichtlich ihrer Gegenstandsadäquanz begründet.

Wenn diese Überlegung hinsichtlich der Dominanz eines Parsonianischen Paradigmas in der Entwicklung der Rational Choice Theorie zutrifft, ist es um so bemerkenswerter, daß Parsons selbst dieses duale Paradigma wenige Jahre nach der „Structure of Social Action“ aufgegeben hat. Ich denke hier an eine Entwicklung, die mit dem 1939 veröffentlichten Aufsatz „The Professions and Social Structure" beginnt (Parsons 1939). Zwei Momente sind auffällig. Erstens entwickelt Parsons eine Kritik am ökonomischen Rationalitätsbegriff nach. Diese beruht im wesentlichen darauf, daß Parsons am Beispiel der Professionen auffällt, daß Rationalität nicht eine basale Begleiterscheinung

\footnotetext{
5iehe dazu interessant Coleman 1990: 260-1.
} 
menschlichen Handelns schlechthin und insofern eine historische Invariante ist, daß Rationalität vielmehr als geschichtsabhängige gesellschaftliche Institution zu verstehen ist, deren moderne Form man u.a. am Beispiel der Institutionalisierung der Professionen ${ }^{6}$ oder auch der amerikanischen oder europäischen Universität ${ }^{7}$ als gesellschaftsgeschichtlich folgenreichen Beispielen studieren kann. Entsprechende Folgerungen hinsichtlich des institutionellen Charakters von Rationalität gelten dann auch für die Einzelhandlung, die sich unter näher anzugebenden gesellschaftlichen Umständen der Erwartung konfrontiert sehen kann, eine rationale Handlung zu sein und daraus folgende Verzichte an Spontaneität, Expressivität, Selbstverwirklichung etc. auf sich zu nehmen. Die Erwartung der Rationalität, der sich die Einzelhandlung gegenübersieht, ist also eine soziale Erwartung, die für die Handlung extern ist. Rationalität wechselt damit bei Parsons auf die Seite der Normen, Werte und Institutionen und deshalb wird der endgültige Übergang zu einer Systemtheorie zwingend, weil jetzt alle Bedingungen des Handelns (inklusive von Rationalität) im System des Handelns ('action frame of reference') zu explizieren sind. Alles andere - Biologie, Physik und die später eingeführte telische Sinnschicht, der sich die Religion verdankt $t^{8}$ - sind Umwelten des Handelns, so daß System/Umwelt als eine universalistische Leitunterscheidung die Unterscheidung Akteur/Situation verdrängt. Eine zweite Umstellung ist die bei Parsons gleichfalls mit der Forschung über Professionen beginnende Entwicklung der „Pattern Variables". Pattern Variables sind fundamentale Orientierungsalternativen des Handelns wie Universalismus vs. Partikularismus, Askription vs. Leistungsorientierung und eben auch Selbstorientierung vs. Kollektivitätsorientierung, die für den Handelnden als Wahlalternativen fungieren. Damit wird deutlich, ohne daß Parsons es je in diesen Worten gesagt hätte, daß jegliches

\footnotetext{
${ }^{6} \mathrm{Vgl}$. dazu mit einer in überraschendem Maße parsonianisch eingefärbten Perspektive Stinchcombe 1990, der an Institutionen vor allem das Moment herausarbeitet, daß die in sie eingebundene Rationalität des Handelns in vielen Fällen der Verwirklichung des Nutzens eines „Anderen" dient (insb. 306, 313-4). Diese Überlegung führt Stinchcombe dann auch dazu, daß er wie der späte Parsons an professionellen Institutionen das treuhänderische ('fiduciary') Moment in der Verwaltung gesellschaftlicher Werte herausstellt.

${ }^{7}$ Vgl. zu diesen Beispielen Parsons/Platt 1973 bzw. Stichweh 1991, ders. 1994

${ }^{x}$ Dazu Parsons 1978.
}

Handeln Unterscheidungsgebrauch ist. Die für die Rational Choice Theorie wichtige Alternative von Selbstinteresse vs. kollektives Interesse ist eine dieser handlungsleitenden Unterscheidungen. Aber sie ist als Unterscheidung eine soziale Vorgabe des Handelns, und es gibt a priori keinen Grund mehr, Selbstinteresse des Handelns als Prämisse unterstellen zu können. In der Theorie soziokultureller Evolution müßte deshalb an die Stelle einer Analyse, die sich fragt, wie man von egoistischen Akteuren zu kollektiven Institutionen kommt, eine Analyse treten, die stattdessen zu klären versucht, wie die Unterscheidung Selbstinteresse vs. Kollektivitätsorientierung historisch entsteht und wie Differenzierungsprozesse je verschiedene Spielräume für jede der beiden Seiten dieser Orientierungsalternative schaffen. Es bilden sich dann in der Gesellschaft Spielräume für selbstbezogenes Handeln, beispielsweise in der Ökonomie Aber dies sind gesellschaftlich institutionalisierte Freiräume, die auf Unterscheidungen aufruhen, die soziohistorisch voraussetzungsreich sind.

\section{Differenztheorien vs. Theorien rationaler Wahl: Die Konstitution rationaler Akteu- re durch Unterscheidungsgebrauch}

Mittlerweile ist die soziologische Systemtheorie in ihrer Entwicklung nach Parsons auf eine Unterscheidungstheorie und zugleich auf eine Kommunikationstheorie umgestellt worden. Dafür haben neben vielen anderen die in den vierziger Jahren entwickelte und zunächst an Maschinen orientierte Informationstheorie von Claude E. Shannon und Warren Weaver (Shannon/Weaver 1949), ihre kommunikations- und differenztheoretische Umformulierung bei Gregory Bateson (Bateson 1973), die den Unterscheidungs- und den Beobachtungsbegriff präzisierende Logik von George Spencer Brown (Spencer Brown 1972) und schließlich die Synthese dieser heterogenen Denkentwicklungen, die Niklas Luhmann vorgelegt hat, ${ }^{y}$ eine entscheidende Rolle gespielt. Kommunikation als die Basisoperation der Gesellschaft ruht dann selbst auf einer Unterscheidung auf, der von Information und Mitteilung, die von einem Beobachter und damit einem 'alter ego' erschlossen wird, das mittels dieser Unterscheidung im Akt des Verstehens eine Kommunikation beobachtet und daran eigene und weitere Kommunikationen anschließen kann. Vor allem aber impliziert Kom-

\footnotetext{
${ }^{9}$ Siehe etwa Luhmann 1990; ders. 1995.
} 
munikation, außer daß sie auf einer sie als Kommunikation definierenden Unterscheidung aufruht, die Handhabung vieler anderer Unterscheidungen, so da $\beta$ fast alles, was für die Rational Choice Theorie wichtig ist, einigermaßen robuste Präferenzen und Interessen von Akteuren, ${ }^{10}$ in der Systemtheorie nur als Unterscheidungsgebrauch rekonstruiert werden kann, wobei es sich in der Regel um Unterscheidungen mit deutlich artikulierter Präferenz für eine der beiden Seiten der Unterscheidung zu handeln scheint. Entsprechendes gilt, wie oben schon gesagt, für Selbstinteresse vs. Kollektivitätsorientierung als eine der Leitunterscheidungen der Rational Choice Theorie.

Wichtig ist dann zunächst einmal folgender Einwand oder folgende Korrektur: Der Akteur und alle von ihm benutzten Unterscheidungen und die Präferenzen, die er innerhalb von Unterscheidungen artikuliert, sind als Resultat von sozialen Konstruktionsprozessen zu sehen. Insofern wirkt die für Rational Choice paradigmatische Formulierung, die den Akteur durch Wahlhandlungen unter institutionellen Constraints beschreibt, eher befremdlich. Bei dem, was im Begriff der Constraints - meinem Eindruck nach auf problematische Weise - zum Verschwinden gebracht wird, handelt es sich um die konstitutiven Bedingungen für die kognitiven Leistungen des Akteurs einschließlich jener Abgrenzungsleistungen, die ihn für sich selbst und für andere überhaupt als einen Akteur identifizierbar machen. Das ist im übrigen ein vertrauter Einwand gegen Rational Choice, den man nicht nur in der Systemtheorie, sondern auch im amerikanischen Neoinstitutionalismus häufig formuliert findet. " Ich will dafür nur zwei Passagen zitieren. Die eine entlehne ich bei DiMaggio und Powell: "Institutions do not just constrain options: they establish the same criteria by which people discover their preferences. In other words, some of the most important sunk costs are cognitive. "12 Eine

\footnotetext{
${ }^{10} \mathrm{Vgl}$. zur Annahme stabiler Präferenzen Stigler/Becker 1977. Bemerkenswert ist bei Stigler und Becker auch, wie abstrakt die Präferenzen (soziale Distinktion, Prestige) gefaßt werden müssen, damit sie als stabil gedacht werden können. Die Soziologie, die dabei her auskommt, unterscheidet sich (auch in der Verwendung des Kapitalbegriffs) nicht sehr von der Pierre Bourdieus.

II Im Rational Choice-Denken selbst scheint sich die Einsicht in diesen Zusammenhang darauf zu konzentrieren. daß konzediert wird, daß Präferenzen aus Aushandlungsprozeduren hervorgehen, während die Konstitution des Akteurs selbst nicht problematisiert wird. Siehe interessant Sen 1995.

12 DiMaggio/Powell 1991: 11; vgl. auch Jaeger 1993: 497-8.
}

andere Formulierung, die ich treffend finde, zitiere ich aus einem Aufsatz von John Meyer. Dieser spricht von dem, fundamental problem of modern social scientific theory: the separation of ideas about exchange from those about the social construction of the units involved in exchange." Angesichts dieser Fehllenkung in der Entwicklung sozialwissenschaftlicher Theorie sei, so Meyer, die kritische institutionalistische Idee zu verfolgen: „that all action simultaneously constructs exchanges and the political world within which these exchanges occur and are given meaning" (Meyer 1987: 4243). Man kann diesen Einwand im übrigen noch ein Stück weitertreiben, indem man ihn nicht nur als Einwand gegen die Vernachlässigung sozialer Konstruktionsprozesse, aus denen Unterscheidungsgebrauch und Präferenzbildung sich allererst ergeben, formuliert. Was darüber hinaus deutlich geworden ist, ist, daß wir es hier mit zirkulären Verhältnissen zu tun haben: Kommunikationen, die Unterscheidungen verwenden, die sie sowohl konstruieren, wie sie andererseits auch behaupten, $\mathrm{da}$ diese Unterscheidungen für sie externe Constraints weiteren Prozessierens sind. Theorien, das sei hier nur en passant notiert, wären auch daran zu messen, wie sie mit zirkulären Verhältnissen dieses Typs umzugehen wissen. Ein gutes Beispiel für einen Lösungsversuch bietet Karl E. Weick in seinem „The Social Psychology of Organizing“ von 1979, das 'enactment' als den Prozeß beschreibt, in dem Organisationen eine Umwelt schaffen, die für sie dann später die letztlich orientierungsrelevante Umwelt bildet. ${ }^{13}$ Wie es in einer der wiederkehrenden Formulierungen Weicks heißt: „People in organizations repeatedly impose what they later claim imposes on them." dus des Umgangs mit Zirkularität fungiert bei Weick Evolutionstheorie, weil sie eine strikte Trennung der Mechanismen und zugleich Beziehungen der Rückkopplung unter Mechanismen zu denken erlaubt.

Eine nächste wichtige Implikation der Umstellung der Systemtheorie auf eine differenz- oder unterscheidungstheoretische Grundbegrifflichkeit betrifft den Rationalitätsbegriff. Wenn Kommunikation und Handeln immer auf Unterscheidungsgebrauch aufruhen und wenn Unterscheidungsge-

\footnotetext{
13 Vgl. Weick 1979: 130: „The term enactment is preferred over variation because it captures the more active role that we presume organizational members play in creating the environments which then impose on them." Vgl. dazu Everett 1994.

14 Weick ebd.: 152.
} 
brauch heißt, daß man Unterscheidungen als Zwei-Seiten-Formen mit Bezeichnung der einen Seite handhabt, ohne gleichzeitig die Einheit der verwendeten Unterscheidung bezeichnen oder gar reflektieren zu können, dann heißt dies auch, daß jeder Unterscheidungsgebrauch sich immer einen blinden Fleck hinsichtlich dieser Einheit der Unterscheidung einhandelt. Die Verwendung der politischen Unterscheidung rechts/links zur Klassifikation politischer Optionen kann nicht uno actu die Kosten der Verwendung dieser Unterscheidung reflektieren. Daraus aber folgt, daß die Rationalitätschancen der einzelnen Kommunikation oder Handlung sehr begrenzt sind. Erst die nächste Kommunikation ist in der Lage, die gerade verwendete Unterscheidung mittels einer anderen Unterscheidung $\mathrm{zu}$ beobachten und derart auch eine Vorstellung darüber zu gewinnen, was man mit der ersten Unterscheidung sieht oder nicht sieht, und erst eine sequentielle Vernetzung vieler solcher Beobachtungen zweiter Ordnung führt auf eine Systembildung hin. Das aber läßt die These der Systemrationalität als der einzigen denkbaren Rationalitätsform plausibel werden und erzeugt zunehmende Distanz zum Rationalitätsbegriff der Rational Choice Theorie, der auf eine unit act-Rationalität oder Handlungsrationalität angewiesen ist. Stattdessen sieht die Systemtheorie Rationalitätschancen allenfalls in der Vernetzung vieler Beobachtungen, die sich auf die Unterscheidungen jeweils früherer Beobachtungen richten, und sie arbeitet am einzelnen kommunikativen Akt eher nichtrationale Momente wie das der Latenz heraus, wobei Latenz aber nicht mehr wie in der europäischen Aufklärung, in der Psychoanalyse oder im Marxismus, korrekturbedürftige Latenz, eine durch Bewußtwerdung zu überwindende Undurchschaubarkeit, vielmehr eine unhintergehbare Latenz hinsichtlich der Einheit einer jeden Unterscheidung als eine Folge des kommunikativen Gebrauchs dieser Unterscheidung meint.

Eine andere Tendenz zur Skepsis hinsichtlich des Rationalitätsbegriffs der Rational Choice Theorien ergibt sich aus einer Fortführung der Parsonianischen Denktradition. Wenn Rationalität eine institutionalisierte Wertorientierung in modernen Gesellschaften ist, dann führt die institutionalisierte Erwartung der Rationalität zu paradoxen Folgen. Das rationale Individuum, der rationale korporative Akteur als eine institutionalisierte Leitidee können gesellschaftsweite Diffusionsprozesse verursachen, aber mit paradoxen Konsequenzen, die bereits Tocqueville identifiziert hat. Die adaptierte Rationalität ist Angleichung und Kopie, die in ihren homogenisierenden Effekten Autonomie untergräbt. ${ }^{15}$ John Meyer und Brian Rowan haben aus dieser Überlegung bekanntlich eine ironische Umdeutung der Weberschen Rationalisierungsthese entwickelt, die an Rationalität in ihrem formalen, kalkulierenden Aspekt das Moment hervorhebt, daß sie in modernen Gesellschaften primär als eine zeremonielle Darstellungsweise wirkt. die auf Außeneffekte berechnet ist, während sie zugleich ein inneres Systemgeschehen verdeckt und legitimiert, das nach anderen Prinzipien organisiert ist. ${ }^{16}$ Insofern Rationalität in diesem Sinn die Innenseite eines Systems verdeckt, würde sie dann doch wieder Autonomie befördern, aber es wäre eine Autonomie, die nach anderen Prinzipien organisiert wäre als denen einer formalen, kalkulierenden Rationalität.

Die Rational Choice Theorie kann auf diese Kritik am Rationalitätsbegriff reagieren, indem sie „Rationalität" als einen differenzlosen Begriff behandelt. Dann wäre Rationalität eine universelle Eigenschaft des menschlichen Erlebens und Handelns und würde in die Nähe von anderen differenzlosen Begriffen rücken, wie sie ja auch die Systemtheorie beispielsweise im Sinnbegriff und im Komplexitätsbegriff kennt. ${ }^{17}$ Die Tendenz zu einer solchen Option ist u.a. im Werk von Herbert Simon lange schon erkennbar. ${ }^{18}$ Als eine Anweisung an den Beobachter ergibt sich damit die folgende: Immer dann, wenn ein menschliches Verhalten Dir als nichtrational oder irrational erscheint, versuche den Rationalitätsbegriff so zu erweitern, daß er auch dieses Verhalten einzuschließen erlaubt! Unter diesen Voraussetzungen entstehen Begriffe wie ,,bounded rationality“ oder ,satisficing behavior". Die Prämisse der Rationalität fungiert offensichtlich als eine Art Suchschematismus, der neue Mechanismen der Informationsverarbeitung

\footnotetext{
15 So Strang/Meyer 1993: 506: „Following Tocqueville, we call attention to the anomalous character of modernity. Its careful analysis of the bounded individual, the rationalized organization, and the purposive society create powerful standardizing forces. The diffusion processes involved directly oppose the internal rationalization sought by the modernizing project and presupposed in its analysis. The modern actors whose uniqueness and autonomy are most celebrated are precisely those most subject to the homogenizing effects of diffusion." Bemerkenswert dazu auch Meyer et al. 1992: 166-7.

${ }^{16}$ Siehe die beiden Aufsätze von Meyer und Rowan in Meyer/Scott 1983: 21-44,71-97; zum Bezug auf Weber vgl. Tyler 1987: 316 .

17 Vgl. Luhmann 1990a.

is Vgl. etwa Simon 1990.
} 
oder Handlungsplanung zu identifizieren versucht, die in den Rationalitätsbegriff inkorporiert werden können und dessen inneren Verweisungsreichtum steigern. Zusätzlich wird dann ein Residualbegriff erforderlich, der die nicht inkorporierbaren Verläufe aufnimmt. Als ein solcher Residualbegriff eignet sich viel eher der Begriff der Irrationalität als der symmetrisch konstruierte und für die Aufnahme von Komplexität gemeinte Pareto/Parsons-Begriff des Nichtrationalen. Jon Elster, der diese Strategie der Weiterentwicklung der Rational Choice Theorie favorisiert, hat die Implikationen präzise erkannt. Die Rational Choice Theorie verliert unter diesen Voraussetzungen ihren prädiktiven Charakter (Elster 1993: insb. 179-180). Sie wird zu einer Hermeneutik des Entscheidungsverhaltens, die, ähnlich wie dies für eine Theorie der Gerechtigkeit gilt, mit bestimmten Intuitionen hinsichtlich dessen beginnt, was Rationalität sein könnte, die aber in der Konfrontation mit realem Entscheidungsverhalten diese Intuitionen unweigerlich modifiziert und insofern den Rationalitätsbegriff als einen ständig veränder- oder erweiterbaren behandeln muß. ${ }^{19}$ Das ist eine interessante Strategie, das Entscheidungsverhalten von individuellen und korporativen Akteuren unablässig daraufhin abzusuchen, welches die in ihm verwirklichte Rationalität sein könnte. Was Elster aber übersieht, der in demselben hier angeführten Aufsatz von 1993 seine Polemik gegen den Funktionsbegriff einmal mehr wiederholt, ${ }^{20}$ ist, daß der Rationalitätsbegriff in der von ihm vorgeschlagenen Fassung in eine exakte Analogie zum Funktionsbegriff der Sozialwissenschaften rückt. Für einen anderen Erklärungsgegenstand, nämlich die Frage der lokalen oder globalen Integration von Komponenten in ein Sozialsystem, schreibt der Funktionsbegriff dieselbe Suchstrategie vor: Bilde zunächst Intuitionen, was die Funktion einer Komponente in einem Sozialsystem sein könnte. Falls sich dies nicht als erfolgreich erweist, suche nach anderen - bis dahin unidentifizierten - Problemen in diesem Sozialsystem, in Hinsicht auf die diese Komponente als Problemlösung fungiert oder früher einmal fungiert hat. Für beide Begriffe - den Rationalitätsbegriff und den Funktionsbegriff dient im übrigen die Evolutionstheorie als Hintergrundtheorie, weil sie Annahmen macht und Mechanismen identifiziert, die zu plausibilisieren er-

\footnotetext{
${ }^{19} \mathrm{Vgl}$. Tversky/Kahneman 1986, die ..Framing“ und „Intransparenz" als Spezifikationen des Simonschen Konzepts der „,bounded rationality“ einführen.

${ }^{20}$ Ebd. 183.
}

lauben, warum ein nichtrationales oder irrationales Verhalten oder eine funktionslose oder dysfunktionale Systemkomponente trotz der in ihnen vorliegenden Nachteile oder Redundanzen über lange Zeiträume überleben können. ${ }^{21}$

\section{James S. Coleman und Niklas Luhmann: Generalisierter Tausch vs. Theorie der Kommunikationsmedien}

Dasselbe Argument, das ich gerade am Beispiel des Rationalitätsbegriffs angedeutet habe, ließe sich für andere Zentralbegriffe der Rational Choice Theorie durchführen. Wie verhält es sich mit „utilities“ oder „Zwecken“ als subjektiven Komponenten eines jeden Handelns. Sind dies differenzlose Begriffe, die etwas bezeichnen, was ein Implikat des Handlungsbegriffs selbst ist, so $\mathrm{da} B$ immer dann, wenn eine Handlung vorliegt, sich ein „Zweck“ oder eine zugehörige „utility" der Handlung zurechnen lä $3 t,{ }^{22}$ und die verbleibende Frage eigentlich nur noch ist, wer diese Zurechnungsentscheidung vornimmt und zu welchem Zeitpunkt des Handlungsablaufs man ihn bittet, dies zu tun. Oder handelt es sich bei „Zweck" und „utility“ um Hermeneutiken des Handelns im Elsterschen Sinne, was die Möglichkeit einschließen würde, $\mathrm{da} ß$ ein Akteur sich ihrer bedient, um sich zu vergewissern oder herauszufinden, welches der Zweck oder die utility seiner Handlung gewesen sein könnte. Im letzteren Fall aber würde man sich erneut die verhaßten „hidden mechanisms“ der funktionalen Analyse einhandeln, die das Selbstverständnis des Akteurs hinsichtlich der Auslegung seiner Handlung erweitern. Gerade diese möchte beispielsweise James S. Coleman vermeiden, wenn er in einem Aufsatz von 1986 die Rational Choice Theorie so vorstellt, daß er sagt, sie ruhe auf einer simultanen Zurückweisung und Akzeptation von Zweckorientierung auf (Coleman 1986): Sie akzeptiere Zwecke auf der individuellen Ebene und weise sie auf der Ebene des Systems zurück. Auf der Ebene des Akteurs aber argumentiere Handlungstheorie funktional: Handlungen würden von ihren antizi-

${ }^{21}$ Zum Zusammenhang von Funktionalismus und Evolutionstheorie siehe etwa Millikan 1984 und Wimsatt 1972. ders. 1986.

22 Dazu interessant Hechter 1994, der ,utility“ als subjektives Element des Handelns so auslegt, daß beliebige "Werte" (also nicht nur individuelle oder instrumentelle Werte) in das Handeln inkorporiert werden können, weil die Tatsache, daß eine Person etwas tut, beweist, daß für sie der korrespondierende Wert „utility“ besitzt. 
pierten Folgen verursacht. Erneut wird die Frage an Coleman sein: Welcher Beobachter ist es, der den Zweck der Handlung (mit welcher Verbindlichkeit) identifiziert und zu welchem Zeitpunkt des Handlungsablaufs tut er dies? ${ }^{23}$

Diese von James $\mathbf{S}$. Coleman vorgelegte Variante der Rational Choice Theorie möchte ich im folgenden etwas näher diskutieren. Coleman hat bekanntlich 1990 nach vielen Vorarbeiten, die in manchen Hinsichten eher der Netzwerk- und der Austauschtheorie zuzurechnen waren, einen umfangreichen Entwurf unter dem Titel „Foundations of Social Theory" publiziert, der vermutlich die soziologisch bisher gehaltreichste Variante des Rational Choice Denkens verkörpert.

Als Ausgangspunkt fungiert erneut ein, wie Coleman es nennt, 'allgemeines Paradigma rationalen Handelns', das unterstellt, daß Akteure „Nutzen“ (,utility“) zu maximieren versuchen und daß sie bei diesem Versuch angebbaren externen Constraints unterworfen sind (Coleman 1990: 152). Den Begriff des Nutzens operationalisiert Coleman für die Zwecke der Sozialtheorie durch den Begriff der „Interessen“. Wenn man eine Fußnote zur historischen Relativität von Theorien dieses Typs machen wollte, könnte man anmerken, daß auch die Aufklärung des späten 18. Jahrhunderts vielfach Theorien über Nutzenorientierung als $\mathrm{Pa}$ radigma für Rationalität entworfen hat, nur mit dem radikal verschiedenen Akzent, daß die rationale Orientierung ('Ausübung des Verstandes'), die auf Nützlichkeit verpflichtet, es dem Akteur gerade erlaubt, sich von der Eingeschlossenheit in das Selbstinteresse zu befreien. ${ }^{24}$

Bei Coleman richten sich Interessen auf Ressourcen oder - und das ist eine ungewöhnliche Option - auf Ereignisse. ${ }^{25}$ Und für beide, Ressourcen und Ereignisse, gilt die duale Struktur, daß Akteure sie entweder bereits kontrollieren oder ein Interesse an ihrer Kontrolle haben. Interesse und Kontrolle können nun aber auseinanderfallen: $d . h$. man kontrolliert Ressourcen und Ereignisse, die einen nicht hinreichend interessieren, und ist andererseits an Ressourcen und Ereignissen sehr interes-

\footnotetext{
${ }^{23}$ Per Implikation ist hier auf die seit Cecil Wright Mills' "Situated Actions and the Vocabulary of Motives" (Mills 1940) extrem umfangreiche Literatur zu verweisen, die mit Bezug auf "Zwecke", "Ziele" etc. von retrospektiven Sinnentwürfen spricht. Siehe statt vieler anderer nur Weick 1979, insb. 195-7; Powell/DiMaggio 1991.

${ }^{24}$ Siehe interessant LaVopa 1988: 191-2, 196, mit Verweisen auf Eckermann, Krünitz, Hartmann.

25 Ausführlich in Coleman 1990, Kap. 2.
}

siert, die man aber nicht kontrolliert. Also muß man sich auf Transaktionen einlassen, in denen Ereignisse und Ressourcen mit anderen Akteuren getauscht werden. Soweit ist im wesentlichen der gemeinsame intellektuelle Kern von Austauschtheorien und Rational Choice Theorien skizziert. Der einzige unkonventionelle Schritt, den Coleman wählt, ist, daß er das, worauf sich Interessen und Kontrollabsichten richten können, um die Kategorie des „Ereignisses" erweitert und damit Handlungen wie Bestechung, Drohungen, Versprechen, Investitionen als Austausch zu analysieren imstande ist, weil das, was in diesen Handlungen getauscht wird, Voraussagen hinsichtlich Ereignissen sind. Im weiteren Argumentationsgang des Buches erweist sich, daß die Ereignisse, an deren Kontrolle Coleman vor allem denkt, Handlungen sind, so daß wir es in den entscheidenden Hinsichten bei Coleman mit einer Austauschtheorie zu tun haben, die den Aufbau komplexer Sozialsysteme aus Tauschvorgängen hervorgehen sieht, in denen Rechte hinsichtlich der Kontrolle von Handlungen getauscht werden. Um dies an einem der einfachsten Sachverhalte zu erläutern: Vertrauen meint für Coleman jene soziale Struktur, in der ich einem anderen die Wahl oder die Kontrolle meiner Handlung überlasse, weil ich unterstelle, daß er dieses Kontrollrecht in meinem Interesse ausüiben wird. ${ }^{26}$

Welches Problem löst die Erweiterung der Austausch- und Rational Choice Theorie um die Kategorie des Ereignisses und die der Kontrolle von Handlungen? Es war immer ersichtlich, daß die Austauschtheorie hinsichtlich dessen beschränkt war, was sie als einen Gegenstand von Tauschhandlungen denken konnte. Richard M Emerson, der bis heute wohl scharfsinnigste Theoretiker der Austauschtheorie, notiert in einem seiner letzten, 1981 publizierten, Aufsätze, daß den Fokus der Austauschtheorie der „Fluß von Benefits“ (,flow of benefits") in Prozessen sozialer Interaktion bilde, während der Informationsfluß in Sozialsystemen primär vom symbolischen Interaktionismus thematisiert werde (Emerson 1981). Parsons, der Intersystembeziehungen bekanntlich als Austausch dachte, hatte dieses Problem durch die $\mathrm{Hy}-$ pothese einer kybernetischen Hierarchie zu lösen versucht, die immer einen Energie- und einen Informationsflu $\beta$ in entgegengesetzte Richtungen postulierte. ${ }^{27}$ Welche Bedeutung kommt in dieser Hinsicht Colemans Vorschlag zu? Ich habe eben

${ }^{26}$ Ebd. Kap. 8.

27 Vgl. dazu Baum/Lechner 1987 
bereits einige Hinsichten angeführt, in denen der Begriff des Austausches erweitert wird, weil es bei Akten wie einem Versprechen oder einer Bestechung um die Kontrolle über Handlungen geht. Weiterhin ist dann bemerkenswert, daß die Um orientierung des Fokus der Austauschtheorie auf den Tausch von Kontrollrechten über Handlungen auf Analysen hinführt, die in der Systemtheorie in der Theorie der Tauschmedien (Parsons) oder der symbolisch generalisierten Kommunikationsmedien (Luhmann) durchgeführt werden. Mit Bezug auf dieses Problem eines generalisierten Tausches, der sich auf Symbole wie Geld oder Status stützt, die als zirkulierende Symbole Dritte in Tauschvorgänge einzubeziehen erlauben, gab es früher schon auffällige Berührungspunkte zwischen Parsons und Coleman, und in Colemans Buch sind viele der faszinierendsten Passagen genau diesem Problemkreis gewidmet: Geld; Status; Vertrauen und schließlich Liebe, die er als den Sachverhalt deutet, daß zwei Individuen sich wechselseitig das Recht über die Kontrolle ihrer Handlungen einräumen ${ }^{28}$ Wichtig sind dann auch bei Coleman klassische Fragestellungen der Theorie der Kommunikationsmedien: die Übertragbarkeit von Versprechen, beispielsweise Zahlungsversprechen, auf Dritte; Banking-Phänomene, die es erlauben, daß viele Individuen einem korporativen Akteur vertrauen und bei diesem entsprechende Kontrollrechte kumulieren und im weiteren dann sowohl konzentrierte Handlungsfähigkeit ermöglichen wie für rapiden Vertrauensentzug anfällig sind.

In kaum einem Argumentationsbereich scheinen Rational Choice Theorie und Systemtheorie so nahe beieinander zu liegen. Andererseits ist die Frage, die an Colemans Theorie zu stellen ist, die, ob er eigentlich weit genug geht, um die u.a. von Emerson benannten Probleme der Austausch- und Rational Choice Theorien zu lösen. Coleman bleibt beim Transaktions- und beim Tauschbegriff stehen, während beispielsweise Luhmann herausarbeitet, daß der Transaktionsbegriff nur einen sehr speziellen Interaktionstyp trifft, der auf der Basis von Wert- oder Bewertungsdifferenzen zwischen Beteiligten auftritt und der Tausch und Konflikt als seine beiden wesentlichen Varianten kennt (Luhmann 1984: 206). Statt des Transaktionsbegriffs benutzt Luhmann den sehr viel allgemeineren Begriff der Übertragung von Selektionsofferten als Grundlage seiner Theorie der Kommunika-

${ }^{28}$ Coleman 1990: 165: vgl. dazu Lindenberg/Frey 1993: 196, die von einer Internalisierung der Utilität des anderen sprechen. tionsmedien. ${ }^{29}$ Eine vielleicht noch folgenreichere Beschränkung in Colemans Theoriebildung ist, daß die Erweiterung des Transaktionsbegriffs, die Coleman vornimmt, nicht über das Moment 'Übertragung der Kontrolle über Handlungen' hinausgeht. Auch hier scheint mir, daß Luhmann mit der Unterscheidung von Handeln und Erleben einen weit konsequenteren Vorschlag entwickelt hat (Luhmann 1978), und wenn man sich diese Unterscheidung vergegenwärtigt, sieht man unmittelbar, daß ein ego einem alter ego nicht nur die Kontrolle über die eigenen Handlungen übertragen kann, daß vielmehr die gleiche Übertragung von Kontrollrechten sich auch auf die Kontrolle über das eigene Erleben beziehen kann. Damit werden schnell große Klassen von Phänomenen sichtbar, die sich Colemans Zugriff entziehen müssen: Wissenschaft, weil eine auf zwingende Weise überzeugende wissenschaftliche Kommunikation zunächst einmal mein Erleben und erst später und in zweiter Instanz mein Forschungshandeln umstrukturiert; Kunst, weil Kunst zwar für denjenigen, um dessen Werke es geht, eine Handlungspraxis ist, sie aber beim Betrachter im günstigsten Fall gelingender Kunstkommunikation eine neue Art des Welterlebens wählt; bemerkenswerterweise gilt dies auch für die Wirtschaft, weil erst die Luhmannsche Perspektive zu sehen erlaubt, daß die spezifische Leistung des Geldes darin besteht, daß Dritte dem Zugriff auf Güter erlebend zusehen können, weil dieser Zugriff über Geld erfolgt und den Dritten ihr eigenes Verfügen über dasselbe symbolisch generalisierte Medium die jederzeitige spätere Möglichkeit eines Zugriffs auf Güter und Dienstleistungen einräumt. Ein interessantes Beispiel ist schließlich auch der Fall der Erziehung. Eine Colemansche Perspektive müßte vermutlich postulieren, daß der Schüler oder das Kind dem Erzieher das Recht auf die Kontrolle über die eigenen Handlungen einräumt, was u.a. heißen würde, daß der Schüler während des Unterrichts in der Schulbank sitzen bleibt, sich am Unterricht zu beteiligen versucht u.ä. Auch in diesem Fall aber scheint das Einräumen der Kontrolle über das Erleben der viel fundamentalere Sachverhalt. Der französische Sozialanthropologe Dan Sperber hat in faszinierenden Analysen wiederholt gezeigt, daß Erziehung gerade auch die Bereitschaft impliziert, rätselhaft scheinende und nahezu unverstandene Wissenskomponenten (beliefs) in das eigene Erleben zu übernehmen (Sperber 1985; ders. 1990). und daß sich der Aufbau kognitiver Komplexität

${ }^{29}$ Siehe zuletzt S. 61-168 in: Luhmann/De Giorgi 1992. 
und die kognitive Toleranz für schwer in die Form von Gewißheit zu überführende Wissensbestandteile (also beispielsweise Religion) genau diesem Mechanismus des Speicherns von unmittelbar noch gar nicht verarbeitbaren Wissensbeständen verdanken. Eine Theorie, die immer in Termini der Kontrolle über Handlungen denkt, ist mit Sachverhalten dieses Typs offensichtlich überlastet.

\section{Mikro/Makro als Leitunterscheidung der Soziologie?}

Eine weitere Überlegung möchte ich an James S. Colemans Theorie anschließen. In seinem Buch wie auch in einer Reihe von Aufsätzen legt er immer wieder großes Gewicht auf das, was er einen Mikro/-Makro-Übergang (micro/macro-transition) nennt und zugleich auf das komplementäre Phänomen des Makro/Mikro-Übergangs. ${ }^{30}$ In der expliziten Modellierung dieser beiden Übergänge sieht Coleman die eigentliche Aufgabe für die Soziologie. Es gibt für ihn allerdings eine zweitbeste Lösung, die an die Stelle eines expliziten Mikro/ Makro-Übergangs die Modellierung der Verhaltensinterdependenz unter Akteuren treten läßt. Es läßt dann aufmerken, daß Coleman einräumen muß, daß fast alle Analysen auf den nahezu tausend Seiten seines Buches von 1990 in Termini von Verhaltensinterdependenz geschrieben sind. Es könnte sein, daß hier ein Ideal postuliert wird, das nicht nur nicht erreichbar ist, sondern vielleicht nicht einmal wünschbar wäre. Natürlich ist die Relevanz von Mikro/Makro-Unterscheidungen unbestreitbar. Die Systemtheorie beispielsweise vermeidet zwar die explizite Benutzung dieser Terminologie, rekonstruiert die Unterscheidung Mikro/ Makro aber in Termini einer Systemebenenklassifikation, in der unter anderem Interaktionssysteme, die in ihrer thematischen Komplexität und zeitlichen Dauer beschränkt sind, von Großsystemen vom Typus der Weltgesellschaft unterschieden werden. Aber bei dieser systemtheoretischen Modellierung werden immer Zusammenhänge zwischen Teilsystemen ein und desselben Gesellschaftssystems untersucht. Coleman hingegen will mit der Mikro/Makro-Terminologie gerade auch die Interdependenzen zwischen Bewußtseinssystemen einerseits (beispielsweise: den Wertpräferenzen oder den Frustrationserfahrungen von Personen) und Strukturumbrüchen in Gesellschaftssyste-

${ }^{30}$ Siehe insb. Coleman 1987; ders. 1990, Kap. 1. men andererseits (z. B.: die Entstehung des Kapitalismus bzw. die Auslösung von Revolutionen) fassen. Das sind Phänomene, die die Systemtheorie in Termini struktureller Kopplung zweier extrem verschiedener und gegeneinander operational geschlossener Systemtypen analysiert. Strukturwahlen im einen Systemtyp können dann Restriktionen für die möglichen Strukturwahlen im anderen Systemtyp nach sich ziehen. Aber dies ist keine Aggregation von Mikroereignissen zu einem Makrogeschehen. Ich möchte hier auf ein Argument verweisen, das Fritz Heider schon 1927 in seinem auch im übrigen genialen Essay „Ding und Medium" vorgetragen hat. Zwei verschiedene GröBenordnungen werden in der Realität, so Heider, fast nie durch Kausalzusammenhänge miteinander verbunden (Heider 1927: 125-128). Heider unterscheidet zwischen dem Schichtenaufbau der Realität, der impliziert, daß die Ereignisse einer Schicht für die Ereignisse einer anderen Schicht nicht wichtig (im Sinne von kausal folgenreich) sind (natürlich fungieren sie als Infrastruktur), und den Kollektivgeschehnissen in einer Schicht, die in gewisser Hinsicht bereits der nächsten Ebene im Schichtenaufbau zugehören. Also wirken nie Individuen einer niederen Ebene unmittelbar auf die nächsthöhere Ebene (es passiert nicht, daß ein Molekül auf ein anderes trifft und als eine Folge ein Schrank auseinanderfällt oder sich auch nur neigt),$^{31}$ es handelt sich immer um die Interaktion zwischen dem Kollektivgeschehen einer Schicht und der nächsthöheren Systemebene. Umgekehrt gilt dann wiederum, daß Kausalität nicht direkt auf die Individuen einer niederen Ebene wirkt, sondern erneut nur auf die Kollektiverscheinungen dieser tieferen Schicht. Natürlich wirft beispielsweise ein Erdbeben Schränke um, und zwar viele oder wenige Schränke. Aber es ist nicht denkbar, daß ein Erdbeben einen bestimmten Schrank selegiert und genau diesen umwirft.

Argumente dieses Typs mögen Zweifel an der Durchführbarkeit und an der heuristischen Fruchtbarkeit der Mikro/Makro-Unterscheidung wecken, wenn man sie mit den Ansprüchen bela-

31 Mittlerweile wird dieser Teil der Heiderschen These durch Chaostheorien relativiert. Andererseits muß man für die Soziologie registrieren, daß das, was die Chaostheorie an unerwarteten Mikro-Makro-Effekten zu postulieren erlaubt, weder in die für Rational Choice typische Aggregationssprache übersetzt werden kann noch es sich dabei um das strategische Einsteuern von Makroeffekten durch rational kalkulierende Mikroeinheiten handeln kann. Zum Verhältnis von Allgemeinheit und Einzigartigkeit in Chaostheorien vgl. interessant Küppers 1993. 
stet, die Coleman ihr zumutet. Natürlich braucht die Soziologie Überlegungen vom Simmel-Typ, die sich dafür interessieren, was in einem Sozialsystem mit N Mitgliedern geschieht, wenn das $\mathbf{N}+1$ te Mitglied hinzutritt und dann im weiteren dafür, welche N's möglicherweise Schwellenübergänge markieren. Aber damit sind wir wiederum innerhalb eines Sozialsystems, und dies sind begrenzte Fragestellungen, für deren Erschöpfbarkeit man auf das Werk von Peter Blau verweisen könnte. $\mathrm{Zu}$ fragen ist, ob die Soziologie nicht im übrigen beispielsweise mit Harrison C. White, einem anderen der produktiven Theoretiker der amerikanischen Soziologie, auf die Überlegung setzen sollte: „scale doesn't matter". Theoretisch interessante Probleme treten, so White, auf verschiedenen Ebenen der Organisation des Sozialsystems in ähnlicher Form auf und das eigentliche Interesse richte sich darauf, sie über Ebenenunterschiede hinweg vergleichend $\mathrm{zu}$ verfolgen. ${ }^{32}$

Beispielsweise ist für eine Theorie der modernen Gesellschaft als Weltgesellschaft die Frage von brennendem Interesse, wie eigentlich heute im einzelnen kommunikativen Akt lokale und globale Momente miteinander interpenetrieren. ${ }^{33}$ Dafür finden sich bei Luhmann oder bei Giddens oder schließlich auch bei Coleman interessante Überlegungen. So beispielsweise in Colemans spannender Hypothese, die großen kollektiven Täuschungen oder kollektiv geteilten Fehleinschätzungen (weltweite Hysterien; spekulative booms hinsichtlich Wertpapieren, die auf weit verbreiteten Fehlinformationen aufruhen) seien eher ein Phänomen des mittleren und späten 19. Jahrhunderts gewesen, das noch relativ wenig Fernkommunikation gekannt habe, so daß Information auf die Verbreitung von Person zu Person angewiesen war (Coleman 1990: 192-193). Wenn diese Diagnose zuträfe, wäre unsere Gegenwart auf der Basis von Fernkommunikation und den in die Fernkommunikation eingebauten Kontrollmechanismen weniger für Massenhysterien anfällig. Ohne hier entscheiden zu wollen, ob dies ein analytisch und empirisch überzeugendes Argument ist, möchte ich notieren, daß gar nicht abzusehen ist, wie man in solchen Phänomenen, in denen Lokales mit Globalem interpenetriert, einen Sortierprozeß ansetzen können sollte, der die betrachteten Komponenten eines Systems auf Mikro und Makro verteilen würde.

\footnotetext{
32 White 1992. Vgl, auch Eccles/White 1986, insb. 141. und die Rezension Abbott 1992.

${ }^{33}$ Dazu näher Stichweh 1995.
}

\section{Theoriendynamik und Interdisziplinarität}

Die vorstehenden Überlegungen haben im Blick auf einige wenige Vergleichsgesichtspunkte die Theoriedynamik der Systemtheorie und der Rational Choice Theorie zu beschreiben und zu vergleichen versucht. Weitere instruktive Vergleichsgesichtspunkte lassen sich unschwer identifizieren, ohne daß wir ihnen hier nachgehen könnten. So gibt es eine auffällige Parallelität in der Entwicklung der Spieltheorie als einem analytischen Instrumentarium, das gerade auch von Rational Choice Theorien benutzt wird, seit den vierziger Jahren und der nahezu gleichzeitig in der systemtheoretischen Soziologie erfolgenden Formulierung und Ausarbeitung des Theorems doppelter Kontingenz, das basale Bedingungen der Entstehung und der fortdauernden Ermöglichung sozialer Systeme zu klären verspricht. ${ }^{34}$ Ein anderer auffälliger Vergleichsgesichtspunkt betrifft die für beide Theorien enge Beziehung zur biologischen Evolutionstheorie: auf der einen Seite die Soziobiologie des Altruismus, die sich dafür interessiert, wie unter extrem individualistischen Prämissen dennoch die Erklärung von Verhaltensweisen möglich ist, die an kollektiven Gütern unter Hintanstellung eigener Interessen orientiert $z u$ sein scheinen; auf der anderen Seite eine Theoriepräferenz, die die biologische Evolutionstheorie als ein Paradigma einer Theorie langfristigen Wandels auffaßt, die kontinuierlichen und diskontinuierlichen Wandel auf das Zusammenwirken einer Mehrzahl untereinander nicht koordinierter evolutionärer Mechanismen zurückzuführen erlaubt. Es zeichnet sich hier ein Gesamtbild für die beiden betrachteten Theorien ab, in dem es einerseits instruktive Punkte der Überschneidung und parallele Problemlagen gibt, die jenseits der den Zugang eher versperrenden polemischen Kontroversen liegen und für die sich der traditionelle wissenschaftstheoretische Apparat, der die bewährten Geschütze „Beschreibung“ und „Erklärung" gegeneinander ins Feld führt, als wenig hilfreich erweist.

Andererseits wird eine Diagnose immer wieder nahegelegt: Rational Choice und Systemtheorie sind hochgradig eigenständige Unternehmungen, die in vielen Hinsichten eher auseinander driften. Wechselseitige Kritik und der Aufweis paralleler Problemlagen beschleunigen die jeweilige Theoriedynamik, aber die Diversität der Theorien wird dadurch um nichts verringert. Daß sich daran auch

34 Parsons 1968: 167-8 registriert diese Koinzidenz. 
in Zukunft trotz der Einbindung beider Unterfangen in ein und dieselbe Disziplin Soziologie nichts ändern wird, dafür läßt sich an erster Stelle vielleicht ein Argument geltend machen. Rational Choice Theorie und Systemtheorie sind jeweils auch - und eben auf radikal verschiedene Weise in interdisziplinäre Milieus eingebettet. Im einen Fall fungieren als ein solches vor allem die Nationalökonomie und die verschiedenen Nachfolgedisziplinen der praktischen Philosophie (social choice, public choice) ${ }^{35} \mathrm{Im}$ anderen Fall, dem der Systemtheorie, sind dies die naturwissenschaftliche, insb. biologische Systemtheorie und die kybernetische und biologische Epistemologie. Die Erhaltung von Diversität in der Disziplin Soziologie wird wesentlich durch diese Verschiedenheit interdisziplinärer Milieus garantiert.

\section{Literatur}

Abbott, A., 1994: Rezension von Harrison C. White, Identity and Control: A Structural Theory of Social Action. Social Forces 72: 895-901.

Bateson, G., 1973: Steps to an Ecology of Mind. Collected Essays in Anthropology. Psychiatry, Evolution and Epistemology. London: Paladin Books.

Baum, R.S. / Lechner, F.J., 1987: Zum Begriff der Hierarchie: Von Luhmann zu Parsons. S. 298-332 in: D. Baecker et al. (Hg.), Theorie als Passion. Niklas Luhmann zum 60. Geburtstag. Frankfurt a.M.: Suhrkamp.

Camic, C., 1989: Structure after 50 Years: The Anatomy of a Charter. American Journal of Sociology 95: 38-107.

Coleman, J.S., 1986: Social Theory, Social Research, and a Theory of Action. American Journal of Sociology 91: 1309-1335.

Coleman, J.S., 1987: Microfoundations and Macrosocial Behavior. S. 153-173 in: J.C. Alexander et al. (Hg.), The Micro-Macro Link. Berkeley: University of California Press.

Coleman, J.S., 1990: Foundations of Social Theory. Cambridge, Mass.: Harvard U.P.

DiMaggio, P.J./ Powell, W.W., 1991: Introduction. S. 1-38 in: W.W. Powell/P.J. DiMaggio (Hg.), The New Institutionalism in Organizational Analysis. Chicago: Chicago U.P.

Eccles, R.G./H.C. White, 1986: Concentration for Control?: Political and Business Evidence. Sociological Forum 1: 131-158.

Elster, J., 1993: Some Unresolved Problems in the Theory of Rational Behavior. Acta Sociologica 36: 179-190.

Emerson, R.M., 1981: Social Exchange Theory. S. 30-65 in: M. Rosenberg/R.H. Turner (Hg.), Social Psychology: Sociological Perspectives. New York: Basic Books.

Everett, J.L., 1994: Communication and Sociocultural Evolution in Organizations and Organizational Populations. Communication Theory 4: 93-110.

\footnotetext{
${ }^{35}$ Siehe dazu interessant Coleman 1990, Sen 1995
}

Hechter, M., 1990: The Emergence of Cooperative Social Institutions. S. 13-33 in: Ders. et al. (Hg.), Social Institutions. Their Emergence, Maintenance and Effects. Berlin und New York: Walter de Gruyter.

Hechter, M., 1994: The Role of Values in Rational Choice Theory. Rationality and Society $6: 318-333$.

Heider, F., 1927: Ding und Medium. Symposion 1: 109 157.

Jaeger, C.C., 1993: The Cultural Evolution of Rational Choice. International Sociology 8: 497-503.

Küppers, B.-O., 1993: Allgemeine Gesetze können die Quelle für Einzigartigkeit sein. Kunstforum 124: 95102.

La Vopa, A.J., 1988: Grace, Talent, and Merit. Poor Students, Clerical Careers, and Professional Ideology in Eighteenth-century Germany. Cambridge: Cambridge U.P.

Lindenberg, S. / Frey, B.S., 1993: Alternatives, Frames, and Relative Prices: A Broader View of Rational Choice Theory. Acta Sociologica 36: 191-205.

Luhmann, N., 1978: Erleben und Handeln. S. 67-80 in: Ders., Soziologische Aufklärung 3. Opladen: Westdeutscher Verlag 1981.

Luhmann, N., 1984: Soziale Systeme: Grundriß einer allgemeinen Theorie. Frankfurt a.M.: Suhrkamp.

Luhmann, N., 1990: Die Wissenschaft der Gesellschaft. Frankfurt am Main: Suhrkamp.

Luhmann, N., 1990a: Haltlose Komplexität. S. 59-76 in: Ders., Soziologische Aufklärung 5. Opladen: Westdeutscher Verlag.

Luhmann, N., 1995: Soziologische Aufklärung 6. Die Soziologie und der Mensch. Opladen: Westdeutscher Verlag.

Luhmann, N. / De Giorgi, R., 1992: Teoria della società. Milano: Franco Angeli.

Meyer, J.M., 1987: The World Polity and the Authority of the Nation State. S. 41-70 in: G.M. Thomas et al., Institutional Structure. Constituting State, Society, and the Individual. Newbury Park: Sage.

Meyer. J.W. et al., 1992: School Knowledge for the Masses: World Models and National Primary Curricular Categories in the Twentieth Century. Washington D.C. und London: Falmer Press.

Meyer, J.W. / Scott, W. R., 1983: Organizational Environments: Ritual and Rationality. Beverly Hills: Sage.

Millikan, R.G., 1984: Language, Thought, and Other Biological Categories. New Foundations for Realism. Cambridge, Mass.: MIT-Press.

Mills, C. W., 1940: Situated Actions and Vocabularies of Motive. American Sociological Review 5: 904-913.

Parsons, T., 1937: The Structure of Social Action. New York: Free Press.

Parsons, T., 1939: The Professions and Social Structure. Social Forces 17: 457-467.

Parsons, T., 1968: Social Interaction. S. 154-176 in: Ders., Social Systems and the Evolution of Action Theory. New York: Free Press 1977.

Parsons, T., 1978: Action Theory and the Human Condition. New York: Free Press. 
Parsons, T. / Platt, G.M., 1973: The American University. Cambridge, Mass: Harvard U.P.

Powell, W.W. / DiMaggio, P.J. (Hg.), 1991: The New Institutionalism in Organizational Analysis. Chicago: Chicago U.P.

Sen, A., 1995: Rationality and Social Choice. American Economic Review 85: 1-24.

Shannon, C.E. / Weaver, W., 1949: The Mathematical Theory of Communication. Urbana Ill., Reprint 1969.

Simon, H.A., 1990: Invariants of Human Behavior. Annual Review of Psychology 41: 1-19.

Spencer Brown, G., 1972: Laws of Form. New York.

Sperber, D., 1985: Anthropology and Psychology: Towards an Epidemiology of Representations. Man 20: 73-89.

Sperber, D., 1990: The Epidemiology of Beliefs. S. 25-43 in: C. Fraser/G. Geskell (Hg.), Psychological Studies of Widespread Beliefs. New York: Oxford U.P.

Stichweh, R., 1980: Rationalität bei Parsons. Zeitschrift für Soziologie 9: 54-78.

Stichweh, R., 1991: Der frühmoderne Staat und die europäische Universität. Zur Interaktion von Politik und Erziehungssystem im Prozeß ihrer Ausdifferenzierung (16.-18. Jahrhundert). Frankfurt a.M.: Suhrkamp.

Stichweh, R., 1994: Die Form der Universität. S. 246-277 in: Ders., Wissenschaft, Universität, Professionen: Soziologische Analysen. Frankfurt a.M.: Suhrkamp.

Stichweh, R., 1995: Zur Theorie der Weltgesellschaft. Soziale Systeme: Zeitschrift für soziologische Theorie 1: $29-45$.
Stigler, G.J. / Becker, G.S., 1977: De Gustibus Non Est Disputandum. S. 191-217 in: K.S. Cook/M. Levi (Hg.), The Limits of Rationality. Chicago: Chicago U.P. 1990.

Stinchcombe, A.L., 1990: Reason and Rationality. S. 285317 in: K.S. Cook/M. Levi (Hg.), The Limits of Rationality. Chicago: University of Chicago Press.

Strang, D. / Meyer, J.W., 1993: Institutional Conditions for Diffusion. Theory and Society 22: 487-511.

Tversky, A. / Kahneman, D., 1986: Rational Choice and the Framing of Decisions. S. 60-89 in: K.S. Cook/M. Levi (Hg.), The Limits of Rationality. Chicago: Chicago U.P. 1990.

Tyler, W., 1987: 'Loosely Coupled' Schools: A Structuralist Critique. British Journal of Sociology of Education 8: 313-326.

Weick, K.E., 1979: The Social Psychology of Organizing. 2. Aufl. Reading, Mass..

White, H.C., 1992: Identity and Control: A Structural Theory of Social Action. Princeton: Princeton U.P.

Wimsatt, W.C., 1972: Teleology and the Logical Structure of Function Statements. Studies in History and Philosophy of Science 3: 1-80.

Wimsatt, W.C., 1986: Heuristics and the Study of Human Behavior. S. 293-314 in: D.W. Fiske/R.A. Shweder (Hg.), Metatheory in Social Science: Pluralism and Subjectivities. Chicago: Chicago U.P. 